\title{
Plasma miRNA expression profiles in rheumatoid arthritis associated interstitial lung disease
}

Shomi Oka ${ }^{1,2}$, Hiroshi Furukawa ${ }^{1,2^{*}}$, Kota Shimada ${ }^{3,4}$, Atsushi Hashimoto ${ }^{3}$, Akiko Komiya ${ }^{2}$, Naoshi Fukui ${ }^{2}$, Naoyuki Tsuchiya ${ }^{1}$ and Shigeto Tohma ${ }^{2}$

\begin{abstract}
Background: Interstitial lung disease (ILD) is frequently associated with rheumatoid arthritis (RA), and is designated RA-associated ILD (RA-ILD). RA-ILD has a large impact on the prognosis of RA. Here, we investigated the micro RNAs (miRNAs) profiles to determine whether they may be useful for diagnosing RA-ILD.

Methods: RNA was isolated from plasma samples and cDNA was synthesized. Real-time RT-PCR analysis was performed to evaluate 752 miRNA expression profiles in plasma pools from RA patients with or without RA-ILD. Sixteen selected miRNA levels were analyzed in individual plasmas from 64 RA patients with or without RA-ILD.

Results: Expression levels of hsa-miR-214-5p (mean relative expression level \pm standard deviation, $8.1 \pm 28.2$ in RA with ILD, $0.2 \pm 0.9$ in RA without ILD, $P=0.0156)$ and hsa-miR-7-5p (56.2 \pm 260.4 in RA with ILD, $4.7 \pm 11.8$ in RA without ILD, $P=0.0362$ ) were higher in RA patients with RA-ILD than in those without. The values of miRNA index $(214,7)$ generated from hsa-miR-214-5p and hsa-miR-7-5p for ILD were significantly elevated in RA patients with RA-ILD compared with those without $(0.122 \pm 0.332$ in RA with ILD, $0.006 \pm 0.013$ in RA without ILD, $P=0.0010)$. The area under the curve value of the receiver operating characteristic curve for the miRNA index $(214,7)$ was 0.740 .
\end{abstract}

Conclusions: To the best of our knowledge, this is the first report of miRNA profiles in RA-ILD. The expression levels of hsa-miR-214-5p and hsa-miR-7-5p were increased in RA with ILD.

Keywords: miRNA profile, Rheumatoid arthritis, Interstitial lung disease, Biological marker

\section{Background}

Rheumatoid arthritis (RA) is a chronic systemic inflammatory autoimmune disease with bone and joint destruction. It is well known that interstitial lung disease (ILD) is frequently associated with RA [1]. Although nonspecific interstitial pneumonia (NSIP) pattern is predominantly observed in collagen disease patients with ILD, usual interstitial pneumonia (UIP) pattern is often observed in RA-associated ILD (RA-ILD) patients [2]. The prognosis of idiopathic interstitial pneumonia patients with UIP pattern was reported to be worse than

\footnotetext{
* Correspondence: furukawa-tky@umin.org

${ }^{1}$ Molecular and Genetic Epidemiology Laboratory, Faculty of Medicine,

University of Tsukuba, 1-1-1 Tennodai, Tsukuba 305-8575, Japan

${ }^{2}$ Clinical Research Center for Allergy and Rheumatology, Sagamihara Hospital, National Hospital Organization, 18-1 Sakuradai, Minami-ku, Sagamihara 252-0392, Japan

Full list of author information is available at the end of the article
}

those with NSIP [3]. The presence of ILD influences the prognosis of RA $[4,5]$. Krebs von den lungen-6 (KL-6) and surfactant protein-D (SP-D) have been used as biomarkers for ILD, but have low sensitivity for the detection of RA-ILD [6, 7]. Thus, better biomarkers for the early screening of RA-ILD are eagerly expected.

Micro RNAs (miRNAs) are small non-coding RNAs with approximate 22 nucleotide length and are stably detected in plasma or serum. It is widely known that miRNAs modulate the expression of protein-coding genes at the post-transcription level and play important roles in cell activation, proliferation, differentiation, or death. Dysregulation of miRNAs in the circulation was detected in the patients with cancer or other diseases and circulating miRNAs could be potential biomarkers for various diseases [8-10]. Some miRNAs in the circulation were also dysregulated in inflammatory diseases, such as RA 
$[11,12]$, inflammatory bowel diseases [13], or idiopathic pulmonary fibrosis (IPF) [14, 15], though the impact of the circulating miRNA in inflammatory diseases does not reach to that in cancer. The expression levels of hsamiR-132, hsa-miR-24, and hsa-miR-125a-5p were altered in plasma from RA patients $[12,16]$. The expression levels of hsa-miR-21 were increased in sera from IPF patients $[14,15,17]$. However, few studies have focused on circulating miRNA profiles of RA-ILD. The present study investigated circulating miRNA profiles of RA-ILD to determine whether they may be useful for diagnosing RA-ILD.

\section{Methods}

\section{Patients}

Sixty four Japanese patients with RA were recruited at Sagamihara Hospital. ILD was diagnosed from computed tomography $(\mathrm{CT})$ findings by two physicians specializing in RA-ILD. RA patients were categorized from A to Z, based on the Sagamihara Criteria [1]. RA cases in categories A to D were RA with ILD $[\operatorname{ILD}(+) R A]$ and those in $\mathrm{G}$ and $\mathrm{H}$ were $\mathrm{RA}$ without $\operatorname{ILD}[\operatorname{ILD}(-) \mathrm{RA}]$. This study included RA cases in categories A [Findings consistent with ILD were observed in high resolution CT (HRCT) images (length of shorter diameter of the lesion was $\geq 2 \mathrm{~cm}$ in a transverse section)] or H [HRCT images were normal] [1]. RA patients with ILD were further diagnosed with one of the two patterns of UIP or NSIP, based on the predominant CT findings: UIP, irregular linear opacities and honeycombing; NSIP, bilateral ground-glass attenuation patterns predominantly in subpleural and basal regions [2, 18, 19]. Plasma samples from the 64 RA patients with or without ILD were collected and these individual plasma samples were analyzed for miRNA expression profiles. Blood samples were taken in tubes with ethylenediaminetetraacetic acid dipotassium salt (Terumo, Tokyo, Japan) and kept in room temperature before separation. Plasma was separated by centrifugation at $1500 \times \mathrm{g}$ for $10 \mathrm{~min}$, and then stored at $-80{ }^{\circ} \mathrm{C}$ until analysis. All patients fulfilled the American College of Rheumatology criteria for RA [20]. This study was reviewed and approved by Sagamihara Hospital Research Ethics Committee and University of Tsukuba Research Ethics Committee. Written informed consent was obtained from all study participants. This study was conducted in accordance with the principles expressed in the Declaration of Helsinki.

\section{MiRNA analysis}

RNA was isolated from $200 \mu \mathrm{l}$ plasma samples using miRCURY RNA Isolation kit Biofluids (Exiqon, Vedbaek, Denmark) and complementary DNA (cDNA) was synthesized with miRCURY LNA Universal cDNA Synthesis kit II (Exiqon). Real-time RT-PCR analysis was performed to evaluate miRNA expression in the plasma pool from $17 \mathrm{RA}$ patients with RA-ILD or the pool from 17 RA patients without ILD, using Human miRNome microRNA PCR Panel I+ II (Exiqon), ExiLENT SYBR Green master mix (Exiqon), and LightCycler 480 System II (Roche, Basel, Switzerland). Thermal cycling conditions consisted of initial denaturation at $95{ }^{\circ} \mathrm{C}$ for $10 \mathrm{~min}$, followed by 45 cycles of $95{ }^{\circ} \mathrm{C}$ for $10 \mathrm{~s}$ followed by $60{ }^{\circ} \mathrm{C}$ for $1 \mathrm{~min}$. Interplate calibration was conducted by the subtraction of the average $\mathrm{CT}_{\mathrm{T}}$ values of UniSP3 probe of the plate. The levels of miRNA among plasma samples were normalized to the average expression of five circulating miRNAs; hsa-miR-425-5p, hsamiR-423-5p, hsa-miR-103a-3p, hsa-miR-191-5p, hsa-miR93-5p. The amount of miRNA was quantified using comparative $\mathrm{C}_{\mathrm{T}}$ method. The two pooled plasma of ILD(+)RA or ILD(-)RA were screened for the miRNA profiling. The data obtained from the microRNA PCR panels were deposited in Gene Expression Omnibus of National Center for Biotechnology Information and are accessible by accession number GSE88899. The PCR panel contains 752 probes for human miRNAs. Potential miRNA markers were selected for real-time RT-PCR analysis of miRNAs in individual patient plasma, based on the PCR panel data; eight miRNAs with higher absolute $\triangle \Delta$ C т values (The $\triangle{ }_{\Delta}$ C т values of these eight miRNAs were higher than 3.12 ) were selected when the samples with undefined Ст values of wells for detection of gene of interest were eliminated for the selection. Other eight miRNAs with higher absolute ${ }_{\triangle} \mathrm{CT}_{\mathrm{T}}$ values (The $\triangle \triangle \mathrm{C}_{\text {T }}$ values of these eight miRNAs were higher than 5.10) were selected when the undefined $C_{T}$ values were substituted by 40 . Thus, sixteen miRNAs were selected for the individual analysis.

Pick-\&-Mix microRNA PCR Panel (Exiqon), ExiLENT SYBR Green master mix (Exiqon), and Applied Biosystems 7500 Fast Real-Time PCR System (Thermo Fisher Scientific, Waltham, MA, USA) were employed for the detection of miRNAs in each individual plasma sample from 64 RA patients with or without RA-ILD that include the above-mentioned $34 \mathrm{RA}$ patients in the screening. The same thermal cycling condition was used. The expression levels of miRNAs in the samples with undefined $\mathrm{C}_{\mathrm{T}}$ values were define to be 0 . The levels of miRNA among plasma samples were normalized to the average expression of three circulating miRNAs; hsamiR-103a-3p, hsa-miR-191-5p, hsa-miR-93-5p. Relative expression levels of miRNAs in plasma were calculated with comparative $\mathrm{C}_{\mathrm{T}}$ method.

\section{Statistical analysis}

Differences in patient characteristics were analyzed by Mann-Whitney's U test or Fisher's exact test using 2X2 contingency tables. Mann-Whitney's $U$ test was conducted for the comparison of miRNA expression levels. Multiple linear regression analysis was performed to 
develop miRNA index for ILD in RA from miRNA levels. Correction for multiple testing was performed by calculating false discovery rate Q-value [21]. Target genes for the miRNAs were predicted using target prediction algorithm at miRDB (http://mirdb.org) [22].

\section{Results}

\section{Clinical features of the RA patients}

Characteristics of the RA patients are shown in Table 1. Mean age, percentage of males, KL-6, SP-D, and disease activity score 28 (DAS28) in ILD(+)RA were higher than in ILD(-)RA. The percentage of administration of biological disease-modifying anti-rheumatic drugs (DMARDs) in $\operatorname{ILD}(+)$ RA were lower than in ILD(-)RA. Mean age, percentage of males, KL-6, and SP-D in RA with ILD of UIP pattern $[\mathrm{UIP}(+) \mathrm{RA}]$ were higher than in ILD(-)RA. The percentage of administration of biological DMARDs in UIP(+)RA were lower than in ILD(-)RA. Mean age, disease duration, KL-6, SP-D, DAS28, and DAS28CRP in RA with ILD of NSIP pattern $[\operatorname{NSIP}(+) R A]$ were higher than in $\operatorname{ILD}(-)$ RA.

\section{MiRNA expression profiles of RA patients with or without ILD}

Pooled plasma miRNA profiles were compared between $\operatorname{ILD}(+)$ RA and ILD(-)RA groups (Additional file 1: Table
S1). Of 752 tested miRNA, 530 miRNAs were upregulated and 217 miRNAs were down-regulated in ILD $(+)$ RA. Sixteen miRNAs with higher absolute $\triangle \triangle \mathrm{CT}$ values were selected for further analysis on the individual plasmas, as described in Materials and methods (indicated in red, Additional file 1: Table S1).

Sixteen miRNAs as potential biomarkers (hsa-miR29c-3p, hsa-miR-154-5p, hsa-miR-543, hsa-miR-214-5p, hsa-miR-382-3p, hsa-let-7g-3p, hsa-miR-9-5p, hsa-miR370-3p, hsa-miR-221-5p, hsa-miR-483-5p, hsa-miR-7-5p, hsa-miR-376b-3p, hsa-miR-487b-3p, hsa-let-7f-1-3p, hsa-miR-500a-5p, hsa-miR-582-5p) were selected for further analysis of miRNAs in individual patient plasma. The 16 miRNAs were also compared between the ILD(+)RA and ILD(-)RA using the real-time RT-PCR method (Table 2 and Additional file 2: Figure S1). The results show a tendency for the expression levels of hsamiR-214-5p and hsa-miR-7-5p to be increased in the ILD $(+)$ RA group (Fig. 1a and b). Although, male percentage is higher in the ILD(+)RA group, the expression levels of 16 selected miRNAs were not different between male and female in the ILD(+)RA group (Additional file 3: Table S2). The effects of the age on the expression levels of some miRNAs were detected (Additional file 4: Table S3). The effects of the DAS28 on the expression

Table 1 Characteristics of the RA patients

\begin{tabular}{|c|c|c|c|c|c|c|c|c|}
\hline & & ILD(+)RA & UIP(+)RA & NSIP $(+) R A$ & ILD(-)RA & $\begin{array}{l}\text { ILD }(+) \mathrm{RA} \\
P\end{array}$ & $\begin{array}{l}\text { UIP }(+) R A \\
P\end{array}$ & $\begin{array}{l}\text { NSIP }(+) R A \\
P\end{array}$ \\
\hline Number & & 32 & 18 & 14 & 32 & & & \\
\hline Age & year & $70.2(7.4)$ & $70.6(8.0)$ & $69.6(6.8)$ & $57.7(13.8)$ & 0.0002 & 0.0013 & 0.0043 \\
\hline male number & n (\%) & $11(34.4)$ & $9(50.0)$ & $2(14.3)$ & $2(6.3)$ & ${ }^{*} 0.0109$ & ${ }^{*} 0.0007$ & $* 0.5745$ \\
\hline Disease duration & year & $15.5(12.6)$ & $12.8(12.2)$ & $18.9(12.7)$ & $10.6(7.4)$ & 0.1830 & 0.8953 & 0.0116 \\
\hline Steinbrocker stage III and IV & n (\%) & $18(56.3)$ & $9(50.0)$ & $9(64.3)$ & $21(65.6)$ & ${ }^{*} 0.6088$ & ${ }^{*} 0.3701$ & $* 1.0000$ \\
\hline Smoker or past smoker & n (\%) & $10(33.3)$ & $7(38.9)$ & $3(25.0)$ & $10(32.3)$ & $* 1.0000$ & ${ }^{*} 0.7581$ & $* 0.7272$ \\
\hline Rheumatoid factor positive & n (\%) & $30(93.8)$ & $17(94.4)$ & $13(92.9)$ & $27(84.4)$ & ${ }^{*} 0.4258$ & ${ }^{*} 0.3991$ & ${ }^{*} 0.6506$ \\
\hline Anti-citrullinated peptide antibody positive & n (\%) & $31(96.9)$ & $18(100.0)$ & $13(92.9)$ & $31(96.9)$ & $* 1.0000$ & *1.0000 & ${ }^{*} 0.5208$ \\
\hline $\mathrm{KL}-6$ & $\mathrm{U} / \mathrm{ml}$ & $1096.4(853.7)$ & $1086.2(828.4)$ & $1108.8(914.9)$ & $315.3(257.9)$ & $2.44 \times 10^{-7}$ & $2.97 \times 10^{-6}$ & 0.0001 \\
\hline SP-D & $\mathrm{ng} / \mathrm{ml}$ & $194.1(244.7)$ & $177.3(192.3)$ & $214.5(303.0)$ & $46.0(37.5)$ & $2.48 \times 10^{-5}$ & 0.0008 & 0.0001 \\
\hline DAS28 & & $4.0(1.2)$ & $3.7(1.3)$ & $4.3(0.9)$ & $3.1(1.2)$ & 0.0040 & 0.0793 & 0.0021 \\
\hline DAS28-CRP & & $3.0(1.3)$ & $2.6(1.4)$ & $3.4(1.0)$ & $2.4(1.0)$ & 0.0500 & 0.5855 & 0.0035 \\
\hline NSAIDs administration & n (\%) & $13(41.9)$ & $7(38.9)$ & $6(46.2)$ & $15(48.4)$ & *0.7989 & *0.5647 & *1.0000 \\
\hline Corticosteroid administration & n (\%) & $26(83.9)$ & $14(77.8)$ & $12(92.3)$ & $25(80.6)$ & $* 1.0000$ & $* 1.0000$ & *0.6542 \\
\hline DMARDs administration & n (\%) & $24(77.4)$ & $14(77.8)$ & $10(76.9)$ & $30(96.8)$ & *0.0529 & ${ }^{*} 0.0542$ & ${ }^{*} 0.0706$ \\
\hline sDMARDs administration & n (\%) & $19(61.3)$ & $11(61.1)$ & $8(61.5)$ & $19(61.3)$ & $* 1.0000$ & $* 1.0000$ & $* 1.0000$ \\
\hline bDMARDs administration & n (\%) & $10(32.3)$ & $5(27.8)$ & $5(38.5)$ & $22(71.0)$ & ${ }^{*} 0.0048$ & *0.0066 & ${ }^{*} 0.0874$ \\
\hline
\end{tabular}

Average values or numbers of each group are shown. Standard deviations or percentages are shown in parenthesis. Differences were tested by Mann-Whitney's $U$ test or Fisher's exact test using $2 \times 2$ contingency tables

$R A$ rheumatoid arthritis, ILD interstitial lung disease, UIP Usual interstitial pneumonia, NSIP Nonspecific interstitial pneumonia, ILD(+)RA RA with ILD, UIP(+)RA RA with ILD of UIP pattern, NSIP(+)RA RA with ILD of NSIP pattern, ILD(-)RA RA without ILD, KL-6 krebs von den lungen-6, SP-D Surfactant protein-D, DAS28 disease activity score 28, NSAIDs non-steroidal anti- inflammatory drug, DMARD disease-modifying anti-rheumatic drug, sDMARD synthetic DMARD, bDMARD biological DMARD

*Fisher's exact test was employed 
Table 2 miRNA profiles of the RA patients with or without ILD

\begin{tabular}{|c|c|c|c|c|c|c|c|c|c|c|}
\hline \multirow[b]{2}{*}{ miRNA } & \multirow[b]{2}{*}{ ILD(+)RA } & \multirow[b]{2}{*}{ UIP(+)RA } & \multirow[b]{2}{*}{$\operatorname{NSIP}(+) R A$} & \multirow[b]{2}{*}{ ILD(-)RA } & \multicolumn{2}{|l|}{ ILD(+)RA } & \multicolumn{2}{|l|}{ UIP(+)RA } & \multicolumn{2}{|c|}{$\operatorname{NSIP}(+) R A$} \\
\hline & & & & & $P$ & $F D R Q$ & P & $F D R Q$ & $P$ & $F D R Q$ \\
\hline hsa-miR-29c-3p & $28.7(92.4)$ & $45.9(121.6)$ & $6.7(10.4)$ & $2.9(2.5)$ & 0.1772 & 0.6025 & 0.0499 & 0.1698 & 0.9334 & 0.9334 \\
\hline hsa-miR-154-5p & $17.9(59.1)$ & $24.6(76.8)$ & $9.3(21.3)$ & $2.0(2.8)$ & 0.9238 & 0.9815 & 0.5331 & 0.7294 & 0.3604 & 0.7416 \\
\hline hsa-miR-543 & $11.0(35.9)$ & $14.8(46.6)$ & $6.1(13.9)$ & $1.4(1.4)$ & 0.4386 & 0.6213 & 0.8633 & 0.9173 & 0.1145 & 0.6099 \\
\hline hsa-miR-214-5p & $8.1(28.2)$ & $13.6(37.0)$ & $1.0(3.2)$ & $0.2(0.9)$ & 0.0156 & 0.1322 & 0.0076 & 0.0643 & 0.1697 & 0.6099 \\
\hline hsa-miR-382-3p & $10.9(41.5)$ & $14.8(52.9)$ & $5.8(19.9)$ & $0.9(1.7)$ & 0.4279 & 0.6213 & 0.9473 & 0.9473 & 0.1794 & 0.6099 \\
\hline hsa-let-7g-3p & $29.1(107.3)$ & $50.3(141.1)$ & $1.9(3.7)$ & $3.3(5.3)$ & 0.6098 & 0.7974 & 0.5578 & 0.7294 & 0.1095 & 0.6099 \\
\hline hsa-miR-9-5p & $5.8(18.5)$ & $9.1(24.2)$ & $1.5(4.2)$ & $0.9(2.6)$ & 0.8510 & 0.9815 & 0.7724 & 0.8753 & 0.4947 & 0.7416 \\
\hline hsa-miR-370-3p & $12.2(57.4)$ & $18.2(76.3)$ & $4.5(11.1)$ & $0.3(0.4)$ & 0.8766 & 0.9815 & 0.3648 & 0.6202 & 0.4734 & 0.7416 \\
\hline hsa-miR-221-5p & $17.1(53.1)$ & $26.6(68.7)$ & $5.0(16.6)$ & $0.7(1.2)$ & 0.3227 & 0.6213 & 0.0461 & 0.1698 & 0.5235 & 0.7416 \\
\hline hsa-miR-483-5p & $80.7(331.8)$ & $130.5(440.8)$ & $16.6(24.4)$ & $14.3(33.9)$ & 0.2827 & 0.6213 & 0.2330 & 0.4951 & 0.6161 & 0.7888 \\
\hline hsa-miR-7-5p & $56.2(260.4)$ & $91.1(347.0)$ & 11.5 (19.9) & $4.7(11.8)$ & 0.0362 & 0.2049 & 0.0275 & 0.1559 & 0.2616 & 0.6571 \\
\hline hsa-miR-376b-3p & $14.6(48.8)$ & $19.0(63.4)$ & $9.0(19.3)$ & $1.1(1.4)$ & 0.3718 & 0.6213 & 0.2621 & 0.4951 & 0.7915 & 0.8751 \\
\hline hsa-miR-487b-3p & $12.7(56.4)$ & $19.1(75.0)$ & $4.4(10.2)$ & $0.5(0.7)$ & 0.2437 & 0.6213 & 0.1181 & 0.2868 & 0.8237 & 0.8751 \\
\hline hsa-let-7f-1-3p & $24.9(114.7)$ & $39.8(153.0)$ & $5.7(9.4)$ & $2.6(2.8)$ & 0.9893 & 0.9893 & 0.6856 & 0.8326 & 0.6496 & 0.7888 \\
\hline hsa-miR-500a-5p & $55.8(230.6)$ & $94.7(305.3)$ & $5.7(12.3)$ & $1.7(4.6)$ & 0.3744 & 0.6213 & 0.4566 & 0.7056 & 0.4823 & 0.7416 \\
\hline hsa-miR-582-5p & $144.8(288.5)$ & $208.4(360.8)$ & $63.0(124.4)$ & $31.5(60.5)$ & 0.0693 & 0.2946 & 0.0705 & 0.1998 & 0.2706 & 0.6571 \\
\hline miRNA Index $(214,7)$ & $0.122(0.332)$ & $0.202(0.430)$ & $0.019(0.034)$ & $0.006(0.013)$ & 0.0010 & 0.0162 & 0.0005 & 0.0093 & 0.0732 & 0.6099 \\
\hline
\end{tabular}

Average values of each group are shown. Standard deviations are shown in parenthesis. Differences were tested by Mann-Whitney's $U$ test. To correct for multiple testing, the false discovery rate $\mathrm{Q}$-value was calculated

$R A$ rheumatoid arthritis, ILD interstitial lung disease, UIP Usual interstitial pneumonia, NSIP Nonspecific interstitial pneumonia, ILD(+)RA RA with ILD, UIP(+)RA RA with ILD of UIP pattern, NSIP(+)RA RA with ILD of NSIP pattern, ILD(-)RA RA without ILD

levels of miRNAs could not be detected in the present study (Additional file 5: Table S4). The effects of the biological DMARDs on the expression levels of miRNA were not found (Additional file 6: Table S5). For hsamiR-214-5p, 135 target genes were predicted and include SMAD4, coding a protein involved in signal transduction of the transforming growth factor $\beta$ [23]. For hsa-miR-7-5p, 434 target genes were predicted and include BLOC1S4. The mutation of Bloc1s4 causes a model of Hermansky-Pudlak syndrome [24].

The area under the curve (AUC) value of the receiver operating characteristic (ROC) curve for hsa-miR-214$5 p$ was 0.634 and that for hsa-miR-7-5p was 0.652 (Fig. 1c). Specificities and sensitivities of these miRNAs were estimated from the ROC curve conditional on the highest Youden's index. The optimized cut-off level of hsa-miR-214-5p was 0.429 with the sensitivity (0.375) and the specificity (0.906). The optimized cut-off level of hsa-miR-7-5p was 5.686 with the sensitivity $(0.469)$ and the specificity (0.875).

An miRNA index $(214,7)$ was generated from the expression levels of hsa-miR-214-5p and hsa-miR-7-5p and evaluated as a marker for ILD(+)RA; miRNA index (214, 7) $=0.0095 \mathrm{X}$ hsa-miR-214-5p $+0.0008 \mathrm{X}$ hsa-miR-7-5p. The miRNA index $(214,7)$ was higher in the $\operatorname{ILD}(+)$ RA group (Fig. 1d, Table 2, $P=0.0010, Q=0.0162$, mean \pm $\mathrm{SD}=0.122 \pm 0.332 \quad$ in $\quad \operatorname{ILD}(+) \mathrm{RA}, \quad 0.006 \pm 0.013$ in
ILD(-)RA). The AUC value of the ROC curve for the miRNA index $(214,7)$ was 0.740 (Fig. 1e). Specificities and sensitivities of miRNA index $(214,7)$ were estimated from the ROC curve conditional on the highest Youden's index. The optimized cut-off level was 0.004 with the sensitivity (0.656) and specificity (0.813). Thus, the plasma miRNA profiles were different between ILD $(+)$ RA and ILD(-)RA groups.

\section{MiRNA expression profiles of RA patients with UIP or NSIP patterns}

The expression levels of the 16 miRNAs were compared between the UIP( + RA and ILD(-)RA. A tendency for the expression levels of hsa-miR-214-5p and hsa-miR-7$5 p$ to be increased in the UIP(+)RA group was also observed. The values of miRNA index $(214,7)$ in the $\mathrm{UIP}(+) \mathrm{RA}$ group was higher $(P=0.0005, Q=0.0093$, mean $\pm \mathrm{SD}=0.202 \pm 0.430$ in $\mathrm{UIP}(+) \mathrm{RA})$. The expression pattern of the 16 miRNAs were also compared between the NSIP(+)RA and ILD(-)RA. No association was observed in the NSIP(+)RA group. Thus, miRNA profiles were also altered in UIP(+)RA groups.

\section{Discussion}

It was reported that plasma miRNA profiles are altered not only in cancerous patients [8-10], but also in patients with inflammatory diseases [11-15]. However, few 


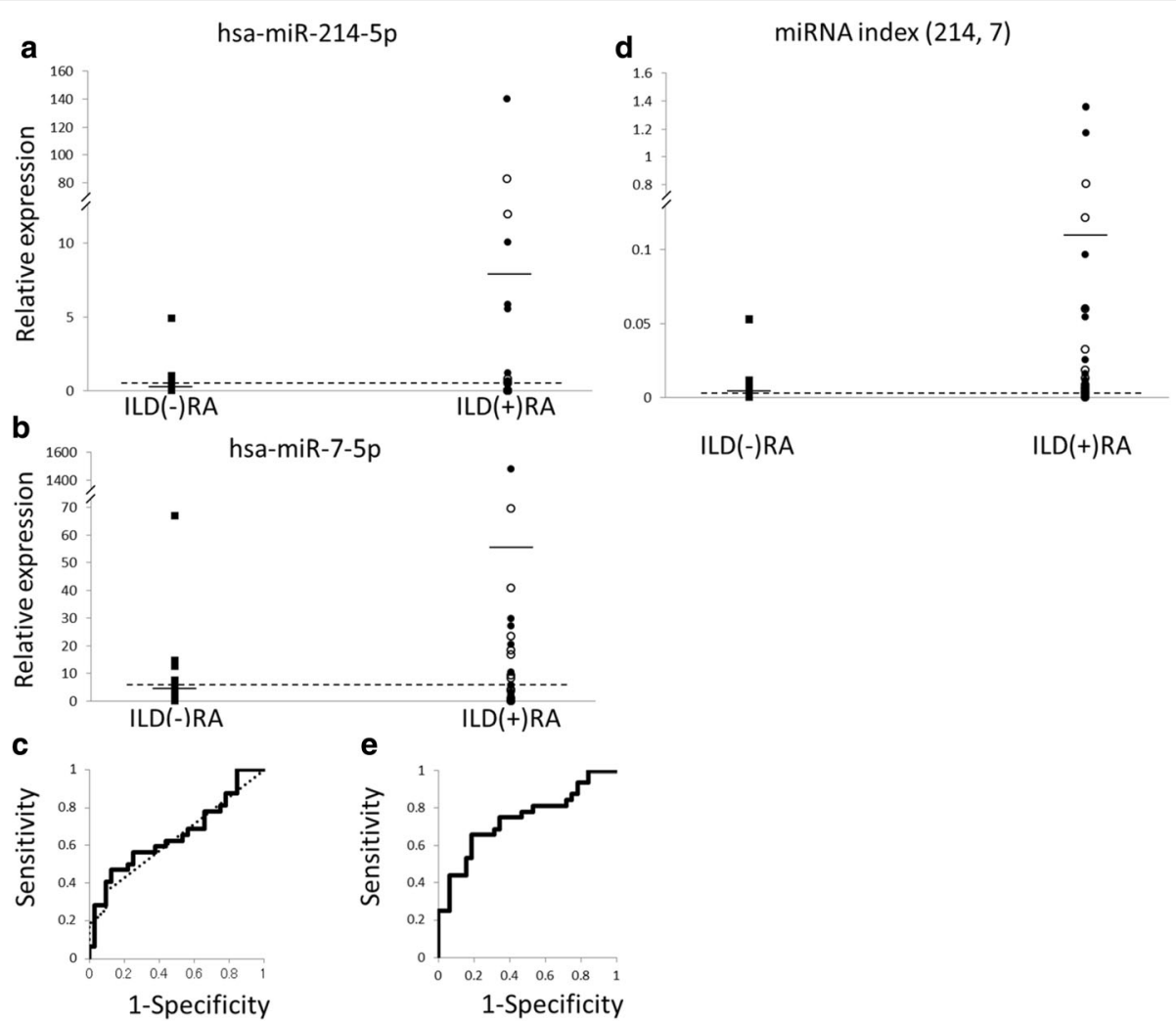

Fig. 1 Evaluation of the miRNA expression levels, as a marker for interstitial lung disease (ILD) in rheumatoid arthritis (RA) patients. a Distribution of the hsa-miR-214-5p. The filled square, filled circle, and empty circle represent RA without ILD, RA with usual interstitial pneumonia, and RA with nonspecific interstitial pneumonia, respectively. Horizontal bars denote means. The horizontal dotted line represents an optimized cut-off level (hsa-miR-214-5p $=0.429$, with specificity and sensitivity of 0.906 and 0.375 , respectively). ILD(+)RA: RA with ILD, ILD(-)RA: RA without ILD. b Distribution of the hsa-miR-7-5p. The horizontal dotted line represents an optimized cut-off level (hsa-miR-7-5p $=5.686$, with specificity and sensitivity of 0.875 and 0.469 , respectively). $\mathbf{c}$ The receiver operating characteristic (ROC) curve using the hsa-miR-214-5p (dotted line) and hsa-miR-7-5p (solid line) as markers for ILD in RA. The area under the curve (AUC) value of the ROC curve for hsa-miR-214-5p is 0.634 and that for hsa-miR-7-5p is 0.652. $\mathbf{d}$ Distribution of miRNA index $(214,7)$ generated from hsa-miR214-5p and hsa-miR-7-5p for ILD (=0.0095 X hsa-miR-214-5p + 0.0008 X hsa-miR-7-5p). The horizontal dotted line represents an optimized cut-off level (miRNA index $(214,7)=0.004$, with specificity and sensitivity of 0.813 and 0.656 , respectively). e The ROC curve using miRNA index $(214,7)$ as a marker for ILD in RA. The AUC value of the ROC curve is 0.740

studies have focused on miRNA profiling in RA-ILD, though these could be diagnostic markers overcoming the existing markers with low sensitivity [6,7]. We have tried to discriminate RA-ILD, one of the potentially lifethreatening extra-articular manifestations of RA, using plasma miRNA profiles. To the best of our knowledge, this is the first report of plasma miRNA profiles in RAILD. We found that hsa-miR-214-5p and hsa-miR-7-5p were increased in the ILD(+)RA group (Fig. 1a and b), though the superiority of these miRNA profiles was not observed compared with KL-6 (AUC $=0.86$, cut-off level $=331.5$, sensitivity $=0.769$, and specificity $=0.842$ ) [7].

It was already well known that cancer cells produce circulating extracellular miRNAs in plasma samples from cancerous patients. Inflammatory cells or tissues may also produce circulating extracellular miRNAs in plasma samples from patients with inflammatory diseases. Thus, hsa-miR-214-5p and hsa-miR-7-5p might be preferentially produced by inflammatory cells or tissues. Though the expression levels of hsa-miR-21 were increased in sera from IPF patients [14, 15, 17], this increase was not observed in the pooled sera from the ILD(+)RA group (Additional file 1: Table S1). This discrepancy could be explained by the difference between the pathogenesis of IPF that includes only UIP and that of RA-ILD that includes both UIP and NSIP.

Since miRNAs modulate the expression of proteincoding genes at the post-transcription level, many studies on the expression and the function of miRNAs were reported. It was reported that the expression levels of hsa-miR-214-5p were increased in B cell lymphoma with better prognosis [25] and liver cirrhosis [26], but decreased in hepatocellular carcinoma [27]. The expression of hsa-miR-7-5p inhibits melanoma cell migration [28], and the decreased expression levels of hsa-miR-7-5p in follicular thyroid cancer were thought to be a reliable 
marker [29]. The expression levels of hsa-miR-7-5p were increased in dental pulp stem cells [30]. The altered expression levels of hsa-miR-7-5p were also reported in breast cancer and glioblastoma [31-33]. Thus, the altered expression patterns and the function of hsa-miR-214-5p and hsamiR-7-5p have been reported. The target genes of the two miRNAs were predicted. Although many genes were predicted, some predicted genes, including SMAD4 and $B L O C 1 S 4$, are known to be involved in the pathogenesis of ILD. However, further functional studies on these miRNAs would be expected to provide better understanding of the roles of these miRNA for the pathogenesis of RA-ILD.

Because of the limited sample size, the validation of miRNA profiles should be performed in future independent work. For the practical applications of miRNA biomarkers for RA-ILD, the expression patterns of all miRNAs should be comprehensively investigated. Therefore, further large-scale miRNA profiling using next generation sequencer could be expected.

\section{Conclusions}

This is the first report of plasma miRNA profiles of RAILD. The expression levels of hsa-miR-214-5p and hsamiR-7-5p were increased in the ILD $(+) R A$ group and they could be potential biomarkers for ILD in RA.

\section{Additional files}

Additional file 1: Table S1. miRNA expression ratios in plasmas from the RA patients with or without ILD. *Undifined CTvalues were substituted by 40 . Selected miRNAs for further analyses were indicated in red (DOCX $82 \mathrm{~kb})$

Additional file 2: Figure S1. Distribution of the miRNA, Krebs von den lungen-6 (KL-6), surfactant protein-D (SP-D), ILDIndex levels, as markers for interstitial lung disease (ILD) in rheumatoid arthritis (RA) patients. The filled square, filled circle, and empty circle represent ILD(-)RA, RA with usual interstitial pneumonia, and RA with nonspecific interstitial pneumonia, respectively. ILD(+)RA: RA with ILD, ILD(-)RA: RA without ILD. (TIFF 687 kb)

Additional file 3: Table S2. miRNA profiles of the RA patients with ILD. Standard deviations are shown in parenthesis. Difference were tested between male and female by Mann-Whitney's $U$ test. RA: rheumatoid arthritis, ILD(+)RA: ILD positive RA. Average values of each group are shown. (DOCX $15 \mathrm{~kb}$ )

Additional file 4: Table S3. miRNA profiles of the RA patients with ILD Average values of each group are shown. Standard deviations are shown in parenthesis. Difference were tested between age $<70$ and age $\geqq 70$ by Mann-Whitney's U test. RA: rheumatoid arthritis, ILD(+)RA: ILD positive RA. (DOCX $15 \mathrm{~kb}$ )

Additional file 5: Table S4. miRNA profiles of the RA patients with ILD. Average values of each group are shown. Standard deviations are shown in parenthesis. Difference were tested between DAS28 $<4.0$ and DAS28 $\geqq 4.0$ by Mann-Whitney's $U$ test. RA: rheumatoid arthritis, ILD(+)RA: ILD positive RA. (DOCX $15 \mathrm{~kb}$ )

Additional file 6: Table S5. miRNA profiles of the RA patients with ILD. Average values of each group are shown. Standard deviations are shown in parenthesis. Difference were tested between bDMARDs $(+)$ and bDMARDs(-) by Mann-Whitney's $U$ test. RA: rheumatoid arthritis, ILD(+)RA: ILD positive RA. (DOCX $15 \mathrm{~kb})$

\section{Abbreviations}

AUC: Area under the curve; cDNA: Complementary DNA; $C$ : Computed tomography; HRCT: High resolution CT; ILD: Interstitial lung disease; ILD(+)RA: RA with ILD; ILD(-)RA: RA without ILD; IPF: Idiopathic pulmonary fibrosis; KL-6: Krebs von den lungen-6; miRNA: Micro RNA; NSIP: Nonspecific interstitial pneumonia; NSIP(+)RA: RA with ILD of NSIP pattern;

RA: Rheumatoid arthritis; RA-ILD: RA-associated ILD; ROC: Receiver operating characteristic; SP-D: Surfactant protein-D; UIP: Usual interstitial pneumonia; UIP(+)RA: RA with ILD of UIP pattern

\section{Acknowledgements}

We thank Ms. Mayumi Yokoyama and Ms. Tomomi Hanawa (Clinical Research Center for Allergy and Rheumatology, Sagamihara Hospital) for secretarial assistance.

\section{Funding}

This study was supported by Grants-in-Aid for Scientific Research (B, C) (26293123, 15K09543), for Young Scientists (B) (24791018) from the Japan Society for the Promotion of Science, Health and Labour Science Research Grants from the Ministry of Health, Labour, and Welfare of Japan, Grants-in-Aid for Practical Research Project for Allergic Diseases and Immunology (Research on Allergic Diseases and Immunology) from Japan Agency for Medical Research and Development, Grants-in-Aid for Clinical Research from National Hospital Organization, Research Grants from Japan Research Foundation for Clinical Pharmacology, Research Grants from Takeda Science Foundation, Research Grants from Daiwa Securities Health Foundation, Research Grants from Mitsui Sumitomo Insurance Welfare Foundation, Research Grants from The Nakatomi Foundation, Bristol-Myers K.K. RA Clinical Investigation Grant from Bristol-Myers Squibb Co., and research grants from the following pharmaceutical companies: Teijin Pharma Limited, Takeda Pharmaceutical Company Limited, Pfizer Japan Inc., Merck Sharp and Dohme Inc., Mitsuibishi Tanabe Pharma Corporation, Eisai Co., Ltd., Chugai Pharmaceutical Co., Ltd., Astellas Pharma Inc., Abbott Japan Co., Ltd. The funders had no role in study design, data collection and analysis, decision to publish, or preparing the manuscript

\section{Availability of data and materials}

All the data were presented in the main text and additional supporting files. The data obtained from the microRNA PCR panels were deposited in Gene Expression Omnibus of National Center for Biotechnology Information and are accessible by accession number GSE88899.

\section{Authors' contributions}

$H F, N T$, and ST conceived and designed the experiments. SO and HF performed the experiments. HF analyzed the data. HF, KS, AH, AK, NF, and ST contributed reagents/materials/analysis tools. SO, HF, NT, and ST wrote the manuscript. All authors read and approved the final manuscript.

\section{Competing interests}

HF has the following conflicts, and the following funders are supported wholly or in part by the indicated pharmaceutical companies; Mitsui Sumitomo Insurance Welfare Foundation was established by Mitsui Sumitomo Insurance Co., Ltd, the Daiwa Securities Health Foundation was established by Daiwa Securities Group Inc., the Takeda Science Foundation is supported by an endowment from Takeda Pharmaceutical Company, the Nakatomi Foundation was established by Hisamitsu Pharmaceutical Co., Inc., and the Japan Research Foundation for Clinical Pharmacology is run by Daiichi Sankyo. HF received honoraria from Ajinomoto Co., Inc., Ayumi Pharmaceutical Corporation, Daiichi Sankyo Co., Ltd., Dainippon Sumitomo Pharma Co., Ltd., Pfizer Japan Inc., Luminex Corporation, and Takeda Pharmaceutical Company. NT is supported by SENSHIN Medical Research Foundation, which is supported by an endowment from Mitsubishi Tanabe Pharma Corporation, and received honoraria from Asahi Kasei Corporation, Eisai Co., Ltd., Daiichi Sankyo Co., Ltd. ST was supported by research grants from pharmaceutical companies: Abbott Japan Co., Ltd., Astellas Pharma Inc., Chugai Pharmaceutical Co., Ltd., Eisai Co., Ltd., Mitsubishi Tanabe Pharma Corporation, Merck Sharp and Dohme Inc., Pfizer Japan Inc., Takeda Pharmaceutical Company Limited, Teijin Pharma Limited. ST received honoraria from Pfizer Japan Inc, Mitsubishi Tanabe Pharma Corporation, Ono Pharmaceutical Co., Ltd., Chugai Pharmaceutical Co., Ltd., AbbVie GK., Astellas Pharma Inc., Asahi Kasei Pharma Corporation. The other authors declare no financial or commercial conflict of interest. 


\section{Consent for publication}

Not applicable.

\section{Ethics approval and consent to participate}

This study was reviewed and approved by Sagamihara Hospital Research Ethics Committee and University of Tsukuba Research Ethics Committee. Written informed consent was obtained from all study participants.

\section{Author details}

${ }^{1}$ Molecular and Genetic Epidemiology Laboratory, Faculty of Medicine, University of Tsukuba, 1-1-1 Tennodai, Tsukuba 305-8575, Japan. ${ }^{2}$ Clinical Research Center for Allergy and Rheumatology, Sagamihara Hospital, National Hospital Organization, 18-1 Sakuradai, Minami-ku, Sagamihara 252-0392, Japan. ${ }^{3}$ Department of Rheumatology, Sagamihara Hospital, National Hospital Organization, 18-1 Sakuradai, Minami-ku, Sagamihara 252-0392, Japan. ${ }^{4}$ Department of Rheumatic Diseases, Tokyo Metropolitan Tama Medical Center, 2-8-29 Musashi-dai, Fuchu 183-8524, Japan.

Received: 7 June 2016 Accepted: 4 January 2017

Published online: 19 January 2017

\section{References}

1. Furukawa H, Oka S, Shimada K, Sugii S, Ohashi J, Matsui T, Ikenaka T, Nakayama H, Hashimoto A, Takaoka H, et al. Association of human leukocyte antigen with interstitial lung disease in rheumatoid arthritis: A protective role for shared epitope. PLoS One. 2012;7(5):e33133.

2. Mori S, Cho I, Koga Y, Sugimoto M. Comparison of pulmonary abnormalities on high-resolution computed tomography in patients with early versus longstanding rheumatoid arthritis. J Rheumatol. 2008;35(8):1513-21.

3. Flaherty KR, Toews GB, Travis WD, Colby TV, Kazerooni EA, Gross BH, Jain A, Strawderman 3rd RL, Paine R, Flint A, et al. Clinical significance of histological classification of idiopathic interstitial pneumonia. Eur Respir J. 2002;19(2):275-83.

4. Turesson C, Jacobsson L, Bergstrom U, Truedsson L, Sturfelt G. Predictors of extra-articular manifestations in rheumatoid arthritis. Scand J Rheumatol. 2000;29(6):358-64.

5. Koduri G, Norton S, Young A, Cox N, Davies P, Devlin J, Dixey J, Gough A, Prouse P, Winfield J, et al. Interstitial lung disease has a poor prognosis in rheumatoid arthritis: results from an inception cohort. Rheumatology. 2010; 49(8):1483-9.

6. Ohnishi H, Yokoyama A, Kondo K, Hamada H, Abe M, Nishimura K, Hiwada K, Kohno N. Comparative study of KL-6, surfactant protein-A, surfactant protein-D, and monocyte chemoattractant protein-1 as serum markers for interstitial lung diseases. Am J Respir Crit Care Med. 2002;165(3):378-81.

7. Furukawa H, Oka S, Takehana K, Muramatsu T, Shimada K, Komiya A, Fukui N, Tsuchiya N, Tohma S. Plasma amino acid profiles in collagen disease patients with interstitial lung disease. Immunome Res. 2013:9(1):1000064.

8. Ng EK, Chong WW, Jin H, Lam EK, Shin WY, Yu J, Poon TC, Ng SS, Sung JJ. Differential expression of microRNAs in plasma of patients with colorectal cancer: a potential marker for colorectal cancer screening. Gut. 2009;58(10):1375-81.

9. Huang Z, Huang D, Ni S, Peng Z, Sheng W, Du X. Plasma microRNAs are promising novel biomarkers for early detection of colorectal cancer. Int $J$ Cancer. 2010;127(1):118-26.

10. Schetter AJ, Okayama H, Harris CC. The role of microRNAs in colorectal cancer. Cancer J. 2012;18(3):244-52.

11. Duroux-Richard I, Jorgensen C, Apparailly F. What do microRNAs mean for rheumatoid arthritis? Arthritis Rheum. 2012;64(1):11-20.

12. Murata K, Furu M, Yoshitomi H, Ishikawa M, Shibuya H, Hashimoto M, Imura Y, Fujii T, Ito H, Mimori T, et al. Comprehensive microRNA analysis identifies miR-24 and miR-125a-5p as plasma biomarkers for rheumatoid arthritis. PLoS One. 2013;8(7):e69118.

13. Wu F, Guo NJ, Tian H, Marohn M, Gearhart S, Bayless TM, Brant SR, Kwon JH Peripheral blood microRNAs distinguish active ulcerative colitis and Crohn's disease. Inflamm Bowel Dis. 2011;17(1):241-50.

14. Li P, Li J, Chen T, Wang H, Chu H, Chang J, Zang W, Wang Y, Ma Y, Du Y, et al. Expression analysis of serum microRNAs in idiopathic pulmonary fibrosis. Int J Mol Med. 2014;33(6):1554-62.

15. Yang G, Yang L, Wang W, Wang J, Xu Z. Discovery and validation of extracellular/circulating microRNAs during idiopathic pulmonary fibrosis disease progression. Gene. 2015;562(1):138-44.
16. Murata K, Yoshitomi H, Tanida S, Ishikawa M, Nishitani K, Ito H, Nakamura T. Plasma and synovial fluid microRNAs as potential biomarkers of rheumatoid arthritis and osteoarthritis. Arthritis Res Ther. 2010;12(3):R86.

17. Li P, Zhao GQ, Chen TF, Chang JX, Wang HQ, Chen SS, Zhang GJ. Serum miR-21 and miR-155 expression in idiopathic pulmonary fibrosis. J Asthma. 2013;50(9):960-4.

18. Tanaka N, Kim JS, Newell JD, Brown KK, Cool CD, Meehan R, Emoto T, Matsumoto T, Lynch DA. Rheumatoid arthritis-related lung diseases: $C T$ findings. Radiology. 2004;232(1):81-91.

19. Oka S, Furukawa H, Shimada K, Sugii S, Hashimoto A, Komiya A, Fukui N, Suda A, Tsunoda S, Ito $S$, et al. Association of human leukocyte antigen alleles with chronic lung diseases in rheumatoid arthritis. Rheumatology (Oxford). 2016. in press.

20. Arnett FC, Edworthy SM, Bloch DA, McShane DJ, Fries JF, Cooper NS, Healey LA, Kaplan SR, Liang MH, Luthra HS, et al. The American Rheumatism Association 1987 revised criteria for the classification of rheumatoid arthritis. Arthritis Rheum. 1988;31(3):315-24.

21. Benjamini Y, Hochberg Y. Controlling the false discovery rate: a practical and powerful aproach to multiple testing. J R Stat Soc B. 1995;57(1):289-300.

22. Wang $X$. Improving microRNA target prediction by modeling with unambiguously identified microRNA-target pairs from CLIP-Ligation studies. Bioinformatics. 2016;2016:6.

23. Shioda T, Lechleider RJ, Dunwoodie SL, Li H, Yahata T, de Caestecker MP, Fenner MH, Roberts AB, Isselbacher KJ. Transcriptional activating activity of Smad4: roles of SMAD hetero-oligomerization and enhancement by an associating transactivator. Proc Natl Acad Sci U S A. 1998;95(17):9785-90.

24. Ciciotte SL, Gwynn B, Moriyama K, Huizing M, Gahl WA, Bonifacino JS, Peters LL. Cappuccino, a mouse model of Hermansky-Pudlak syndrome, encodes a novel protein that is part of the pallidin-muted complex (BLOC-1). Blood. 2003;101(11):4402-7.

25. Lim EL, Trinh DL, Scott DW, Chu A, Krzywinski M, Zhao Y, Robertson AG, Mungall AJ, Schein J, Boyle M, et al. Comprehensive miRNA sequence analysis reveals survival differences in diffuse large B-cell lymphoma patients. Genome Biol. 2015;16(18):18.

26. lizuka M, Ogawa T, Enomoto M, Motoyama H, Yoshizato K, Ikeda K, Kawada $\mathrm{N}$. Induction of microRNA-214-5p in human and rodent liver fibrosis. Fibrogenesis Tissue Repair. 2012;5(1):12.

27. Wojcicka A, Swierniak M, Kornasiewicz O, Gierlikowski W, Maciag M, Kolanowska M, Kotlarek M, Gornicka B, Koperski L, Niewinski G, et al. Next generation sequencing reveals microRNA isoforms in liver cirrhosis and hepatocellular carcinoma. Int J Biochem Cell Biol. 2014;53:208-17.

28. Giles KM, Brown RA, Epis MR, Kalinowski FC, Leedman PJ. miRNA-7-5p inhibits melanoma cell migration and invasion. Biochem Biophys Res Commun. 2013;430(2):706-10.

29. Stokowy T, Wojtas B, Fujarewicz K, Jarzab B, Eszlinger M, Paschke R. miRNAs with the potential to distinguish follicular thyroid carcinomas from benign follicular thyroid tumors: results of a meta-analysis. Horm Metab Res. 2013; 46(3):171-80.

30. Vasanthan P, Govindasamy V, Gnanasegaran N, Kunasekaran W, Musa S, Abu Kasim NH. Differential expression of basal microRNAs' patterns in human dental pulp stem cells. J Cell Mol Med. 2015;19(3):566-80.

31. Calvano Filho CM, Calvano-Mendes DC, Carvalho KC, Maciel GA, Ricci MD, Torres AP, Filassi JR, Baracat EC. Triple-negative and luminal A breast tumors: differential expression of miR-18a-5p, miR-17-5p, and miR-20a-5p. Tumour Biol. 2014;35(8):7733-41.

32. Liu Z, Liu Y, Li L, Xu Z, Bi B, Wang Y, Li JY. MiR-7-5p is frequently downregulated in glioblastoma microvasculature and inhibits vascular endothelial cell proliferation by targeting RAF1. Tumour Biol. 2014;35(10):10177-84.

33. Dong L, Li Y, Han C, Wang $X$, She L, Zhang H. miRNA microarray reveals specific expression in the peripheral blood of glioblastoma patients. Int J Oncol. 2014;45(2):746-56. 